\title{
Vacancy-enhanced intermixing in highly strained InGaAs/GaAs multiple quantum well photodetector
}

\author{
Alex S. W. Lee and E. Herbert Li ${ }^{\text {a) }}$ \\ Department of Electrical and Electronic Engineering, University of Hong Kong, \\ Pokfulam Road, Hong Kong \\ Gamani Karunasiri \\ Department of Electrical Engineering, National University of Singapore, Singapore 119260
}

(Received 22 October 1998; accepted for publication 6 May 1999)

\begin{abstract}
Impurity-free vacancy disordering techniques using rapid thermal annealing with electron-beam evaporated $\mathrm{SiO}_{2}$ encapsulant was utilized to study its effect on the optical and electrical properties of the highly strained InGaAs/GaAs quantum well infrared photodetector. The photoluminescence peak is blueshifted and its line width does not increase much, indicating the compositional disordering of the quantum well structure and there is no strain relaxation or minimal deterioration of the heterostructure quality. Both transverse electric and transverse magnetic infrared intersubband transitions are retained and observed after intermixing. The absorption peak wavelength is redshifted from the as grown $10.2 \mu \mathrm{m}$ to the interdiffused 10.5 and $11.2 \mu \mathrm{m}$, for 5 and $10 \mathrm{~s}$ annealing at $850{ }^{\circ} \mathrm{C}$, respectively, without appreciable degradation in absorption strength. Theoretical calculations of the absorption spectra are in good agreement with the experimental data. Annealed responsivity spectra of both $0^{\circ}$ and $90^{\circ}$ polarization are of comparable amplitude but with narrower spectra line width. Dark current of the annealed devices is found to be an order of magnitude larger than the as-grown one at 77 K. (C) 1999 American Institute of Physics.
\end{abstract}

[S0021-8979(99)04416-3]

\section{INTRODUCTION}

The study of long wavelength quantum well infrared photodetector (QWIP) based on intersubband transitions (ITs) has attracted much research interest and progressed rapidly ${ }^{1-8}$ since the first experimental demonstration of large dipole moment in AlGaAs/GaAs multiple quantum well (MQW). ${ }^{9}$ However, most of the studies have been limited to an AlGaAs/GaAs MQW system owing to the mature growth and processing technology. Because of the polarization selection rule, only incident light with a component of the electric field parallel to the growth direction ( $z$ axis) can be absorbed in a $n$-type QW. Normal incidence is possible only with other optical coupling schemes. ${ }^{10}$ With the development of strained layer QW and band gap engineering, a high quality pseudomorphic QW is achievable. It has been demonstrated that normal incident strained InGaAS/GaAs QWIP ${ }^{11}$ are possible without grating coupling. Due to the smaller effective mass and the higher electron mobility, $n$-typed InGaAs/GaAs QWIPs offer better infrared detector properties than $\mathrm{AlGaAs} / \mathrm{GaAs}$ material. But the thermal stability of strained layers, when subjected to heat treatment, is of prime importance in photonic integrated circuits applications, especially for structures with a higher In concentration. This is because the critical layer thickness is smaller in highly strained heterostructure and there is an increased risk of strain relaxation by the generation of misfit dislocation during thermal annealing.

\footnotetext{
${ }^{\text {a)} E l e c t r o n i c ~ m a i l: ~ e h l i @ e e e . h k u . h k ~}$
}

QW intermixing has attracted increasing attention recently as a useful tool not only for spatially selective tuning of the QW band gap, but also as a means to monolithically integrate devices of differing functionality on a single substrate without requiring additional lithography. ${ }^{12}$ Postgrowth tuning of the AlGaAs/GaAs IT absorption peak wavelength ${ }^{13}$ and QWIP detection wavelength range ${ }^{14,15}$ have also been demonstrated by this technology. In this article, the influence of layers interdiffusion on the performance of a highly strained $n$-doped $\mathrm{In}_{0.3} \mathrm{Ga}_{0.7} \mathrm{As} / \mathrm{GaAs} \mathrm{QWIP}$ at $850^{\circ} \mathrm{C}$ is studied. We demonstrate in our experiments that the QWIP absorption wavelength can be tuned to a redshift of $\sim 1 \mu \mathrm{m}$ and the heterostructure quality is not deteriorated by means of impurity-free vacancy disordering. Both the TE and TM intersubband transitions are observed after intermixing and the responsivity of these annealed devices is comparable to the as-grown device. Theoretical calculation of the absorption spectra, taking into account the interdiffusion and strain effects, is shown to be in good agreement with the experimental results. We also show that the dark current characteristics and the carriers transport properties under an applied electric field have been modified after interdiffusion.

\section{EXPERIMENT}

The QWIP samples used in this study were grown by molecular beam epitaxy (MBE) on a (100) semi-insulating GaAs substrate. The MQW consists of 50 periods of $40 \AA$ wide $\operatorname{In}_{0.3} \mathrm{Ga}_{0.7}$ As wells and 300 - $\AA$-thick undoped GaAs barrier layers. The wells were doped with $\mathrm{Si}$ to a density of about $2 \times 10^{18} \mathrm{~cm}^{-3}$. The top and bottom of the MQW had 
$n^{+}$GaAs cap $(0.5 \mu \mathrm{m})$ and buffer $(1 \mu \mathrm{m})$ layers, respectively, for ohmic contacts. Each QW was designed to have only one bound state inside the well and the first excited state in the continuum above the barrier. The energy difference between the first two eigenstates approximately corresponds to wavelength $\cong 10 \mu \mathrm{m}(\hbar \omega=124 \mathrm{meV})$. Prior to annealing process, approximately a 250 -nm-thick $\mathrm{SiO}_{2}$ dielectric layer was deposited on the samples surface using an electron-beam evaporator. Rapid thermal annealing (RTA) was carried out using a halogen lamp annealing system (AST SHS 10) with double strip graphite heater in nitrogen ambient. During annealing, the sample surfaces were protected from surface degradation by means of a GaAs proximity cap. One of the samples was annealed for anneal time $t_{A}=5 \mathrm{~s}$ at $850{ }^{\circ} \mathrm{C}$ while the other for $t_{A}=10 \mathrm{~s}$ at the same temperature. Mesa diode $\left(200 \times 200 \mu \mathrm{m}^{2}\right)$ were fabricated using standard lithographic technique and wet chemical etching. AuGe/Ni/Au alloyed ohmic contacts were made to the top and bottom contact layers of the device.

\section{THEORETICAL MODEL}

The interdiffusion process in this model is based on the thermal induced intermixing of $\mathrm{Ga}$ and $\mathrm{In}$ atoms in the InGaAs/GaAs MQW. The diffusion of group III atoms in the model is described by the Fick's law with equal and constant diffusion coefficients ${ }^{16}$ in both the well and barrier layers. The extent of interdiffusion is characterized by the diffusion length $L_{d}=(D t)^{1 / 2}$, where $D$ is diffusion coefficient and $t$ is diffusion time. The In composition profile, $w(z)$, as a function of $L_{d}$ is given by

$$
w(z)=\frac{w_{0}}{2}\left\{\operatorname{erf}\left[\frac{L_{z}+2 z}{4 L_{d}}\right]+\operatorname{erf}\left[\frac{L_{z}-2 z}{4 L_{d}}\right]\right\}
$$

where $w_{0}$ is the as-grown In mole fraction, $z$ denotes the coordinate along the crystal growth direction, $L_{z}$ is the asgrown well width, and $\operatorname{erf}(y)$ denotes the error function. From the diffused In composition profile defined by Eq. (1), interdiffused QW parameters can be calculated separately, including band gap, $E_{g}(z)=E_{g}[w=w(z)]$, well-barrier discontinuity, $\Delta E_{g}(z)=E_{g}(z)-E_{g}(z=0)$, and well depth, $V_{c}(z)=Q_{c} \Delta E_{g}(z)$, where the subscript $C$ denotes the electron in the conduction band and $Q_{c}$ is the conduction band offset. To account for the effect of strain, the interdiffusion induced QW confinement profile, $U_{c}(z)$, is defined as: $U_{c}(z)=Q_{c}\left[E_{g}(z)-E_{g}(z=0)\right]-S_{\perp c}(z), \quad$ where $S_{\perp c}(z)$ $=Q_{c} S_{\perp}(z)$. The change in the bulk band gap, $S_{\perp}(z)$, due to the biaxial component of strain is given by $S_{\perp}(z)$ $=-2 a\left[\left(c_{11}-c_{12}\right) / c_{11}\right] \epsilon,{ }^{17}$ where $a$ is the hydrostatic deformation potential, $c_{i j}$ 's are the elastic stiffness, and $\epsilon$ is the in-plane strain. With the material parameters listed in Table I and the interdiffused QW parameters obtained earlier, the energies and envelope functions of the electron subband edge at the zone center of the Brillouin zone, and the intersubband absorption are calculated based on the models described in Ref. 25. The diffusion coefficient $D$ is determined by fitting the calculated intersubband transition energies with
TABLE I. List of parameters used in the numerical calculation. Note that the value of $D$ and $\Gamma$ are fitting parameters.

\begin{tabular}{lc}
\hline \hline & $\mathrm{In}_{x} \mathrm{Ga}_{1-x} \mathrm{As} / \mathrm{GaAs}$ \\
\hline$E_{g}(\mathrm{eV})$ & $1.425-1.501 x+.436 x^{2}$ \\
$m_{c} / m_{0}$ & $0.0632-0.0419 x$ \\
$m_{h h} / m_{0}$ & $0.5-0.09 x$ \\
$m_{l h} / m_{0}$ & $0.088-0.064 x$ \\
$C_{11}\left(10^{11} \mathrm{dyn} / \mathrm{cm}^{2}\right)$ & $11.9-3.571 x$ \\
$C_{12}\left(10^{11} \mathrm{dyn} / \mathrm{cm}^{2}\right)$ & $5.38-0.854 x$ \\
$T(\mathrm{~K})$ & 300 \\
$D\left(\mathrm{~cm}^{2} / \mathrm{s}\right)$ & $1.3 \times 10^{-15}$ \\
$\Gamma(\mathrm{eV})$ & 0.028 \\
$Q_{c}$ & 0.7 \\
\hline \hline
\end{tabular}

the measured energy shift of the absorption spectra in Fig. 2. It gives a value of $D=1.3 \times 10^{-15} \mathrm{~cm}^{2} \mathrm{~s}^{-1}$ to an error within $0.1 \mathrm{meV}$.

\section{RESULT AND DISCUSSION}

Photoluminescence (PL) measurements were performed at $4.5 \mathrm{~K}$ using the $514.5 \mathrm{~nm}$ argon laser at a power of 200 $\mathrm{mW}$ prior to and following the annealing. Figure 1 shows the PL spectra of the as-grown and annealed MQW. The PL peak shifts progressively to higher energy with anneal time from as-grown 1.316-1.319 and $1.323 \mathrm{eV}$, respectively. The blueshift of the band gap energy indicates the intermixing of group III elements near the heterostructure interfaces and the changes in the ground state energy of both the conduction and valence bands. The peak intensity of the $t_{A}=5 \mathrm{~s}$ sample is increased by nearly one fold in magnitude, while that of the $t_{A}=10 \mathrm{~s}$ sample is decreased by almost the same order, with respect to the as-grown sample. The increase in the peak luminescent intensity may be due to the removal of nonradiative centers from the InGaAs/GaAs interfaces. And the reduction in intensity with successive intermixing is most likely due to the defects that act as trapping centers introduced during the annealing process. The full width at half maximum (FWHM) PL line width of the annealed samples does not increase much as compare to the as-grown sample;

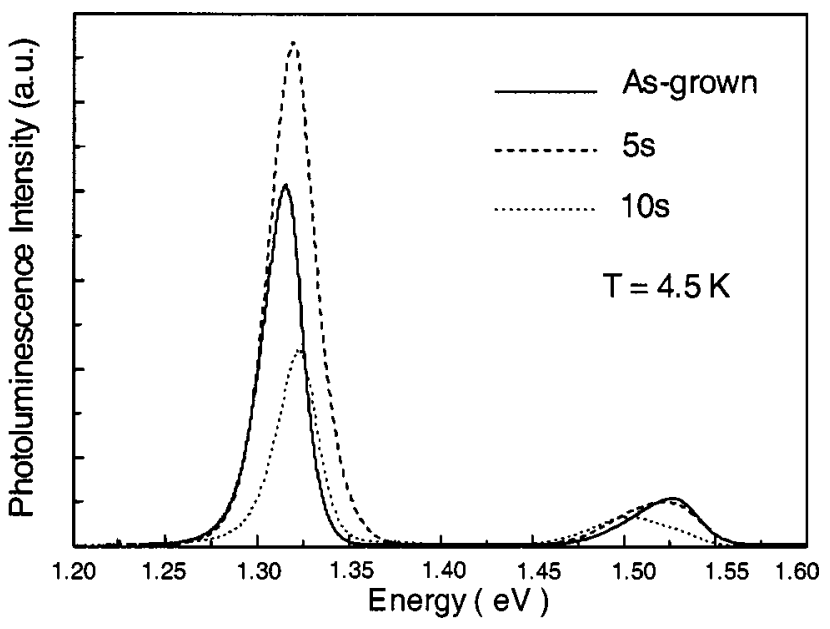

FIG. 1. PL spectra of the as-grown, 5 and $10 \mathrm{~s}$ interdiffused InGaAs/GaAs MQW at $T=4.5 \mathrm{~K}$. 


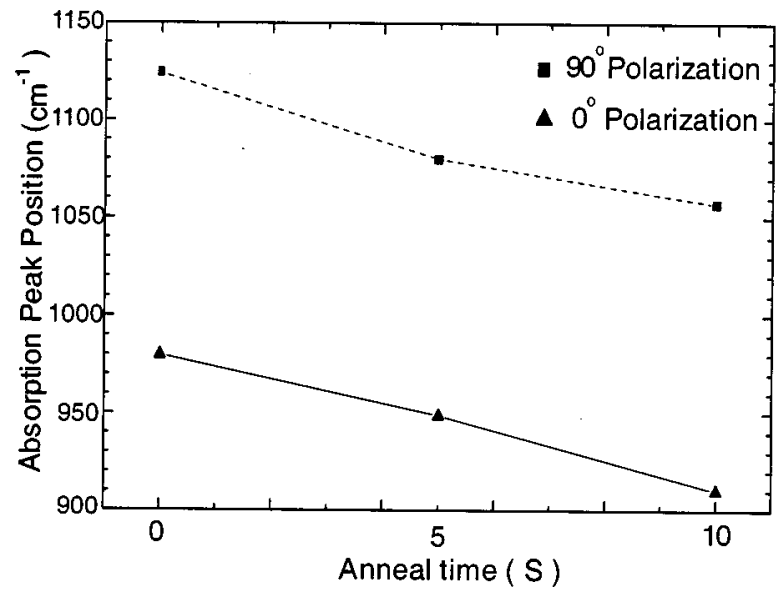

FIG. 2. The redshift of the absorption peak as a function of annealing time for $0^{\circ}$ and $90^{\circ}$ polarizations. The lines are an aid to the eye.

about 4 and $1 \mathrm{meV}$, for the 5 and $10 \mathrm{~s}$ annealed samples, respectively. Since the well width is below the critical thickness for $30 \%$ In concentration, the small variation in PL FWHM may indicate that the onset of strain relaxation or misfit dislocation formation has not been initiated and that there may even be a recovery of strain or an improvement in the heterostructural quality after RTA. ${ }^{18,19}$ The PL peak broadening for the shorter annealed sample is believed due to the native defects near the surface incorporated during growth. Peaks were also observed at about $1.5 \mathrm{eV}$, which are redshifted with interdiffusion, in contrast to the PL peaks observed earlier. These peaks may be due to the luminescence from the GaAs either in the top cap layer or the bottom buffer layer of the MQW.

Intersubband absorption measurement was taken using Nicolet Magna-IR 850 Fourier transform infrared spectrometer at room temperature with a $45^{\circ}$ polished multipass waveguide geometry. The spatial resolution of this technique is about $4 \mathrm{~cm}^{-1}$. Effect of interdiffusion on the intersubband optical absorption of annealed QWs is evidenced in Fig. 2 where the as-grown absorption peak is redshifted linearly with anneal time for both polarizations. For $0^{\circ}$ angle polarization, i.e., a mixture of TE and TM polarizations that contains a component of photon electric field along the growth (z) direction as well as a component in the plane of the layers, the absorption peak is shifted from the as-grown 980 $\mathrm{cm}^{-1}$ (at $10.2 \mu \mathrm{m}$ ) to 949 (at $\left.10.5 \mu \mathrm{m}\right)$ and $910 \mathrm{~cm}^{-1}(11.2$ $\mu \mathrm{m})$, for the 5 and $10 \mathrm{~s}$ annealed samples, respectively. Whereas for normal incident (TE) absorption (the result of band-mixing effects induced by the coupling between the conduction and valence band and is usually forbidden in conventional polarization selection rule), ${ }^{20}$ the absorption peak is shifted with increasing $t_{A}$ from $1124 \mathrm{~cm}^{-1}$ (at $8.9 \mu \mathrm{m}$ ) to $1080\left(\right.$ at $9.26 \mu \mathrm{m}$ ) and $1057 \mathrm{~cm}^{-1}$ (at $9.46 \mu \mathrm{m}$ ), respectively. The difference in the peak wavelength between the two polarizations is due to the $D_{2 d}$ tetragonal perturbation of the local crystal and strain field effects on the QW. ${ }^{21}$ The redshift of the absorption peaks may indicate the subband ground-state energy $E_{1}$ and the first excited-state energy $E_{2}$ (at which oscillator strength, $f$, is a maximum) are being modified (for e.g., $E_{1}$ being pushed higher or $E_{2}$ being

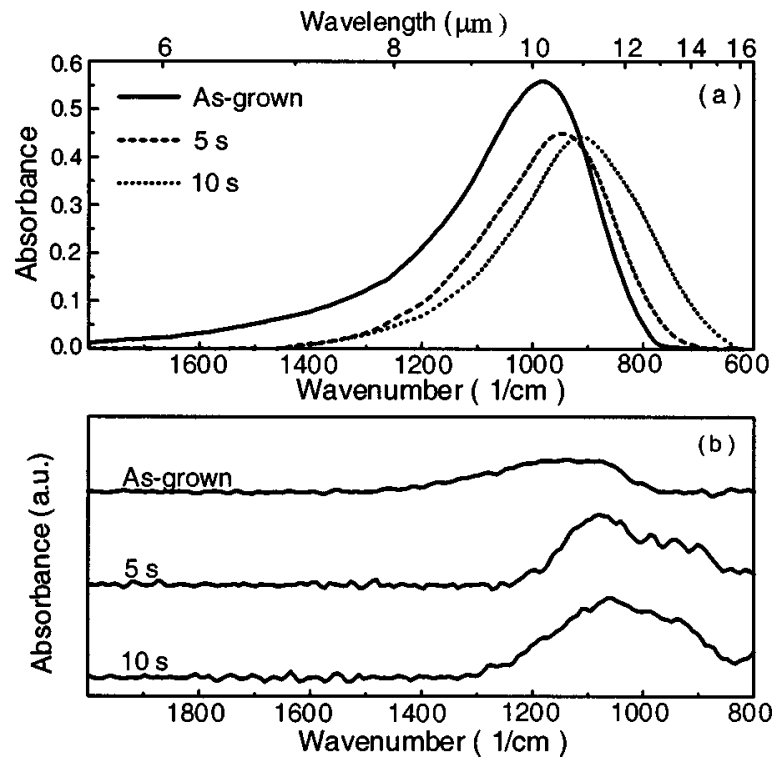

FIG. 3. Absorption spectra of the as-grown, 5 and $10 \mathrm{~s}$ annealed samples at $300 \mathrm{~K}$ as a function of wavelength for (a) $0^{\circ}(\mathrm{TE}+\mathrm{TM})$ polarization and (b) $90^{\circ}$ (TE) polarizations. (The curves have been shifted vertically for clarity.)

pulled closer to the barrier height or both happen simultaneously) and/or the interdiffusion-induced changes in the doping-dependent depolarization shift, ${ }^{9}$ which result in the postgrowth tuning of the absorption wavelength.

Note in Fig. 3(a) that for $0^{\circ}$ polarization, the annealed absorption spectra reduce in amplitude and undergo broadening with increasing anneal time. Also shown in Fig. 3 [curve (b)] is the absorption spectra at $90^{\circ}$ polarization. The annealed spectra also broaden in shape even though its amplitude cannot be compared directly after the curves are being shifted vertically for clarity. It is known that $\mathrm{Ga}$ is very soluble in $\mathrm{SiO}_{2}$ at an elevated temperature. As intermixing process proceeds, an increase in the concentration of group III vacancies in the QW is expected due to the diffusion of $\mathrm{Ga}$ into the $\mathrm{SiO}_{2}$ dielectric layer. This will in turn increase the dopant $(\mathrm{Si})$ diffusion rate across the heterointerfaces to the undoped GaAs barrier and the encapsulant layer, as a consequence of Fermi-level effect. ${ }^{22}$ The out diffusion of $\mathrm{Si}$ across the interfaces during interdiffusion process not only reduces the free carrier concentration but also enhances layers intermixing. Since absorption coefficient $\alpha(h \omega) \propto \rho_{s}\left(\rho_{s}\right.$ is the two dimension electron density in the well), the reduction in the number of free carrier available to be excited by the incident IR radiation may render to a reduction in the absorbance. In addition to the reduction in carrier density, the impurity scattering together with the modification in subband structure give rise to the broadening and decrease in amplitude of the absorption spectra. But $E_{2}$ remains in the continuum under different annealed conditions produced here, as can be seen from the high-energy tail and the asymmetry of all the absorption spectra shown in Fig. 3(a), which are the characteristic features of bound-to-continuum intersubband transition in QWs. ${ }^{23}$ Theoretical calculation of the normalized absorption spectra are shown in Fig. 4 (where broadening factor $\Gamma=28,29$, and $31 \mathrm{meV}$ are adopted for the as-grown, $t_{A}=5$ and $10 \mathrm{~s}$ samples), where the absorption 


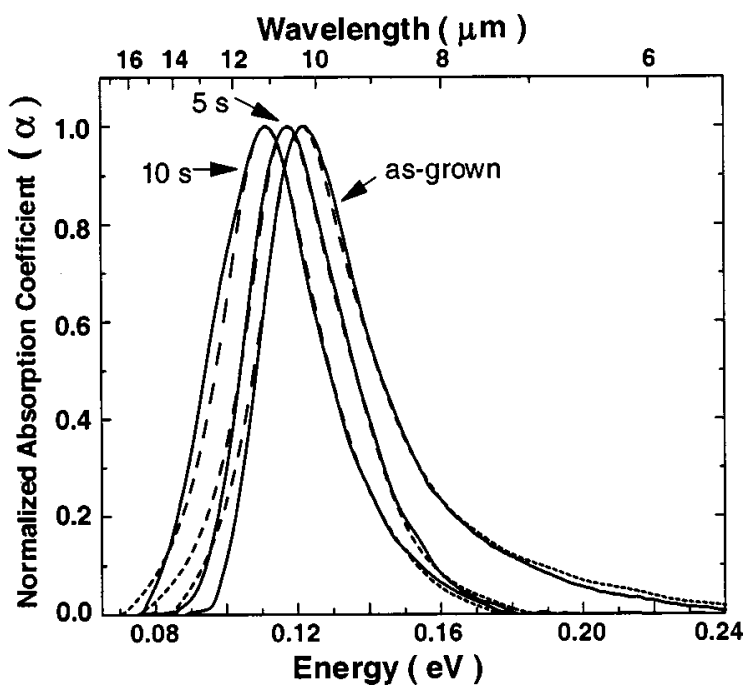

FIG. 4. Normalized absorption spectra of the as-grown, 5 and $10 \mathrm{~s}$ annealed samples (from right to left) at $300 \mathrm{~K}$ as a function of wavelength (solid line: experimental results, dotted line: calculated results).

peak, spectra width, and spectra shapes are in good agreement with the measured results.

Leakage current was measured with the sample mounted on a cold finger at $77 \mathrm{~K}$ using 4156A Parameter Analyzer. The $(I-V)$ characteristic is shown in Fig. 5. Note the asymmetry of the $I-V$ curves between the two polarities. For the as-grown sample, leakage current is higher in reverse bias (i.e., mesa top negative) than in forward bias, which is attributed to the diffusion of dopant species to the growth front during growth or the asymmetrical MBE growth between the GaAs and $\mathrm{In}_{0.3} \mathrm{Ga}_{0.7}$ As interfaces. ${ }^{23,24}$ In contrast to this, the annealed devices exhibit an opposite trend, where the leakage currents are higher at forward bias, and they intersect and overlap with each other in reverse bias. We attribute this to the difference in diffusion rate of In and Ga species across the MQW interfaces, which results in lower barrier height $\mathrm{t}^{25}$ seen by the thermally excited electrons, and the redistribu-

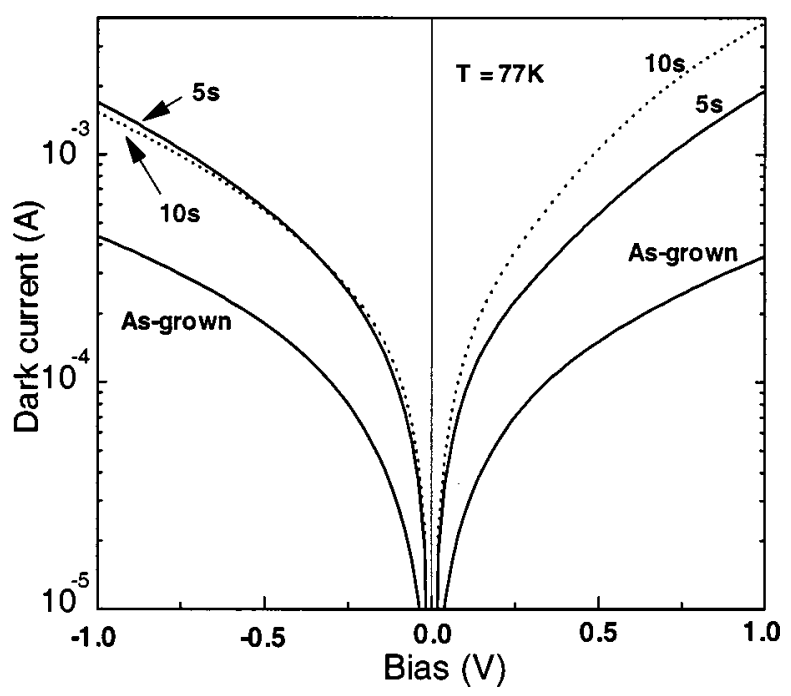

FIG. 5. $I-V$ curve of the as-grown and annealed samples at $T=77 \mathrm{~K}$ as a function of bias.

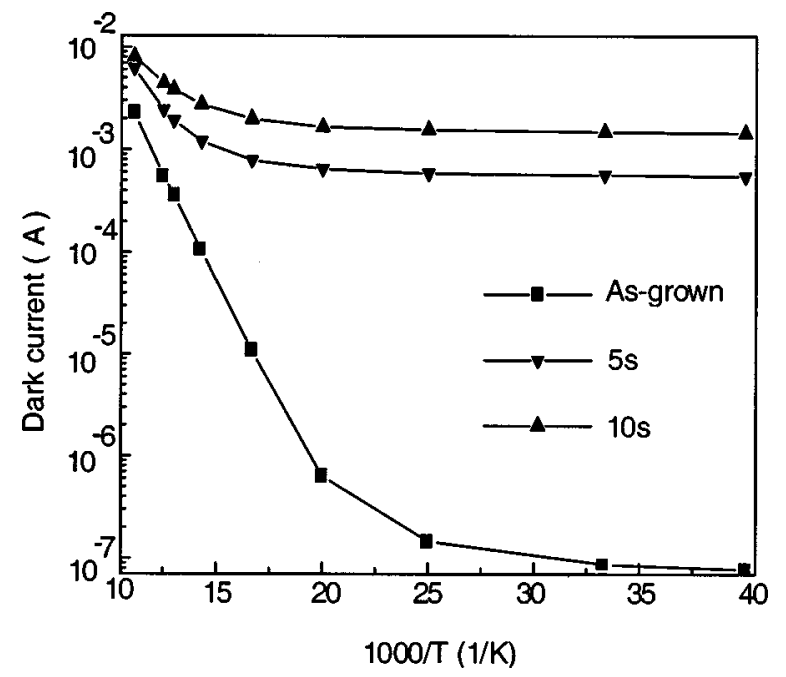

FIG. 6. Leakage current at $V=1 \mathrm{~V}$ as a function of the reciprocal of temperature in the range between 25 and $90 \mathrm{~K}$. The lines are an aid to the eye.

tion of dopant impurity as described earlier. These two factors together with the thinning of the $300 \AA$ barrier not only result in the asymmetry $I-V$ curves, but also give rise to nearly an order of magnitude higher in leakage current at 77 $\mathrm{K}$, as a consequence of annealing.

Figure 6 shows the leakage currents as a function of the reciprocal of temperature. It is interesting to note that the annealed leakage currents are not very sensitive to temperature variation. The leakage currents remain approximately constant for $T<50 \mathrm{~K}$ and increase linearly with higher temperature. Note also that the leakage current of the annealed devices at $T<50 \mathrm{~K}$ is 4 or more orders of magnitude larger than the as-grown sample. At $T>50 \mathrm{~K}$ where thermionic emission mechanism is dominant, all leakage currents increase linearly to almost the same magnitude at $T=90 \mathrm{~K}$. For temperature range from $T=25-90 \mathrm{~K}$, the overall dark current is increased by nearly one order of magnitude and by a factor of 8 , for the $t_{A}=5$ and $10 \mathrm{~s}$ samples, respectively; while the as-grown sample is increased by more than 5 orders of magnitude. Since the leakage current at low temperature is mainly dominated by a tunneling mechanism, the huge increase in leakage current at $T<50 \mathrm{~K}$ is probably related to the defect assisted tunneling. ${ }^{26}$ This is most likely due to the intermixing of $\mathrm{Si}$ and group III constituent atoms in the heterostructure ${ }^{22}$ which introduce defects (group III vacancies) and dopant impurity into the barrier and result in significant increase of electron tunneling through the defect states in the barrier. A further demonstration of this is shown in Fig. 7 where the leakage currents of the annealed samples are very sensitive to bias even at a low temperature $T$ $=25 \mathrm{~K}$. The dark current of the $t_{A}=5 \mathrm{~s}$ sample increases more rapidly at bias $\left|V_{b}\right|>170 \mathrm{mV}$; while the $10 \mathrm{~s}$ annealed device increases at a even higher slope, with the leakage current intersecting and surpassing that of the $t_{A}=5 \mathrm{~s}$ sample at $\left|V_{b}\right| \cong 370 \mathrm{mV}$. But the as-grown leakage current remains flattened throughout the range of bias considered.

The photocurrent was measured using grating monochromator and glowbar source with lock-in detection. A $45^{\circ}$ facet was polished at one end of the device to couple the IR 


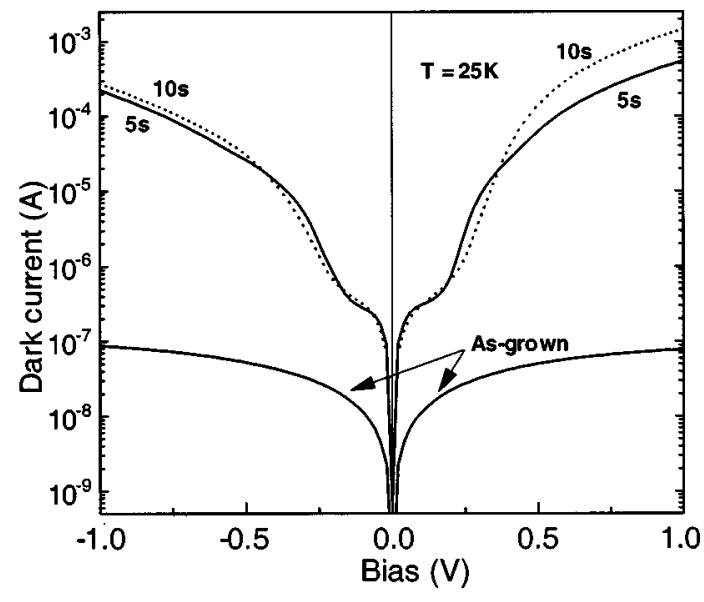

FIG. 7. $I-V$ curve of the as-grown and annealed samples at $T=25 \mathrm{~K}$ as a function of bias.

radiation into the detector structures. ${ }^{27} \mathrm{~A}$ polarizer was inserted in front of the glowbar source in order to study the polarization dependence of the photoresponse. Figure 8 shows the response spectra for $0^{\circ}$ and $90^{\circ}$ polarizations as a function of wavelength at $25 \mathrm{~K}$. The responsivity peaks are redshifted in both spectra, independent of polarization, with respect to the as-grown spectrum. Note in the as-grown spectra that a few satellite peaks appear at rather identical wavelength positions in both as-grown spectra. Since there is only one bound state in the well and the excited state is above the barrier in the continuum, these satellite peaks are most probably due to the intersubband transition from $E_{1}$ to other excited states in the continuum ${ }^{28}$ or to the interaction between $E_{2}$ and other states in the continuum. ${ }^{29}$ However, all these peaks are subdued, as shown in Fig. 8, except for the designed transition peaks that appear in the annealed spectra following RTA. We attribute this to the intermixing induced modification of the QW subband energy states both in the well and the continuum.

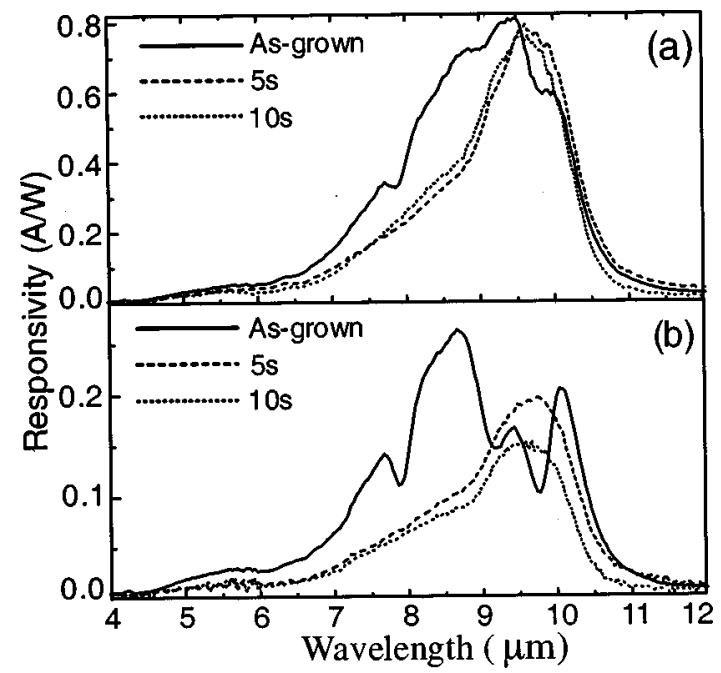

FIG. 8. Photoresponse spectra at $25 \mathrm{~K}$ of the as-grown, 5 and $10 \mathrm{~s}$ annealed samples bias at $2.5,1.05$, and $1.65 \mathrm{~V}$ for (a) $0^{\circ}$ polarization and (b) $90^{\circ}$ polarization, as a function of wavelength.
Figure 8 (a) shows the measurement results for $0^{\circ}$ polarized responsivity at $T=25 \mathrm{~K}$. The responsivity amplitudes are $0.8,0.79$, and $0.77 \mathrm{~A} / \mathrm{W}$, respectively, for the as-grown $(0 \mathrm{~s})$ and annealed $\left(t_{A}=5\right.$ and $\left.10 \mathrm{~s}\right)$ detectors. The three spectra are of comparable magnitude except that the FWHM of the annealed spectra is smaller than the as-grown spectrum, as intersubband transitions to other continuum states are no longer prominent in the annealed spectra. Note that all the spectra have almost identical rising edge. This is as expected since the MQW properties and its structure have not been substantially modified or deteriorated after interdiffusion. The extent of intermixing may not have lowered the barrier height considerably such that those carriers being photoexcited to the extended state above the barrier "see" approximately the same height and are ready to be collected as photocurrent. It is known that the discrepancy of peak position between absorption and responsivity spectra is either due to the increase of barrier height when the temperature is lowered ${ }^{30}$ or the applied electric field. ${ }^{31}$ However, unlike the annealed absorption spectra, the peak positions of the annealed photoresponse do not differ significantly at both polarizations. This phenomenon is probably due to the results of different extents of intermixing on the band structure, polarization effect, low temperature influence, and difference in applied bias. Further work is required to clarify this phenomenon. As the polarization angle is rotated from $0^{\circ}$ to $90^{\circ}$, four pronounced response peaks are observed in the as-grown spectrum of Fig. 8(b). It is also interesting to note that, in contrast to $0^{\circ}$ polarization, the continuum resonances are more dominant in the as-grown spectrum with the dominant peak response occurring at a shorter but not the designed wavelength. Each individual photoresponse spectrum exhibits a different cutoff wavelength under normal incidence illumination, as illustrated in Fig. 8(b). However, this phenomenon is not obvious in the annealed spectra where the photoresponse peak redshifts and locates at a wavelength position similar to that at $0^{\circ}$ polarization. Note also that the responsivity of the $t_{A}=5 \mathrm{~s}$ sample has a larger amplitude than the as-grown and $t_{A}=10 \mathrm{~s}$ samples in the wavelength range of interest between 9 and $10 \mu \mathrm{m}$.

The bias dependence of the responsivity at $0^{\circ}$ polarization for both polarities is shown in Fig. 9. Interestingly, the photoresponse also shows a pronounced asymmetry similar to the $I-V$ characteristic, i.e., the as-grown device has larger responsivity at negative bias while the annealed responsivities have larger magnitude at positive bias, with respect to the reversal of polarity. This indicates that both the photoexcited and nonphotoexcited carriers have similar escape direction under bias and that the transport characteristic is modified after interdiffusion. Since interdiffusion will introduce vacancies and dopand $(\mathrm{Si})$, which act as a kind of defect, into both the barrier and the well, when an electric field is applied to collect the photoexcited carriers, some of the carriers may be trapped by this kind of defect. This may have resulted in the poorer collect efficiency of the interdiffused QWIP and, therefore, lower responsivity under different biases. The responsivity versus bias is quite linear for the three responses, which is an indication of another typical behavior for a bound-to-continuum QWIP. ${ }^{32}$ This is because no tunneling is 


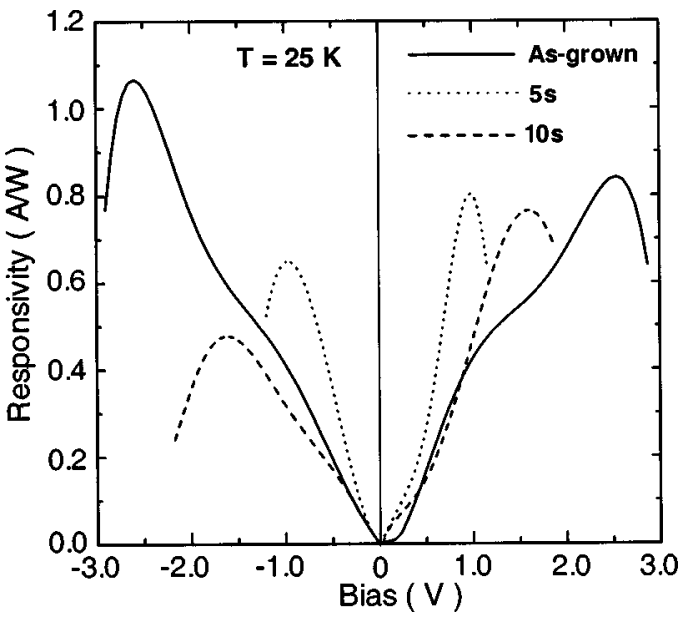

FIG. 9. Bias dependent responsivity of as-grown (solid line), 5 s annealed (dotted line), and $10 \mathrm{~s}$ annealed (dash line) samples as a function of bias at $0^{\circ}$ polarization.

required and the photoexcited carriers that transport above the barrier have a relatively high mobility. The annealed responsivities increase with bias and reach a maximum at bias smaller than the as-grown device (the $5 \mathrm{~s}$ annealed response saturates and decreases at the lowest bias for both polarities), after which they strongly decrease with bias. Since the band structure is modified after intermixing, this is most probably due to the intervalley scattering ${ }^{33}$ where the electrons in the $\Gamma$ valley scatter into the $L$ or $X$ valley minima under an applied electric field, as a result of interdiffusion.

\section{CONCLUSION}

In conclusion, high In composition pseudomorphic interdiffused InGaAs/GaAs QWIP using dopant-enhanced interdiffusion has been demonstrated for its post-growth tunability. PL measurement results show that no strain relaxation or deterioration in structure quality is observed in the highly strained MQW heterostructure at $850{ }^{\circ} \mathrm{C}$. The TE polarization IR intersubband transition, as a result of the bandmixing effects, is preserved, indicating that interdiffusion preserves the intrinsic optical characteristic of the QWIP material. With the modification of subband structure after RTA, both $0^{\circ}$ and $90^{\circ}$ polarizations annealed absorption peaks are redshifted with respect to the as-grown sample without much degradation in absorption strength. Photoresponse peaks due to resonances in the continuum states are subdued after interdiffusion. The annealed photoresponse spectra for $0^{\circ}$ polarization are comparable to the as-grown device with a narrower FWHM, while the designed photoresponse peak becomes dominant at $90^{\circ}$ polarization following RTA. The $I-V$ characteristic of the annealed devices is about an order higher in amplitude than the as-grown one at $77 \mathrm{~K}$ due to the defect-assisted tunneling and is less sensitive to the variation in temperature range from 25 to $90 \mathrm{~K}$. We believe the annealed leakage current can be lowered by optimizing the QWIP structure, such as with thinker barrier, to impede the tunneling mechanism. Thus, interdiffusion is shown to be a useful tool for its ability to fine tune the various aspects of photodetector's performance for grating-free normal incident broadband and multicolor IR applications.

\section{ACKNOWLEDGMENTS}

This work is supported in part by the CRCG grant of the University of Hong Kong, Research Grant CouncilEarmarked Grants of Hong Kong, and National Science and Technology Board under Grant No. GR 6471. The authors would like to thank Prof. S. J. Chua for valuable suggestions, T. Mei, and Dr. S. J. Xu for technical assistance.

${ }^{1}$ S. D. Gunapala et al., IEEE Trans. Electron Devices 44, 1 (1997).

${ }^{2}$ C. J. Chen, K. K. Choi, W. H. Chang, and D. C. Tsui, Appl. Phys. Lett. 71, 3045 (1997).

${ }^{3}$ Y. S. Park, J. Korean Phys. Soc. 32, 443 (1998).

${ }^{4}$ S. D. Gunapala, B. F. Levine, L. Pfeiffer, and K. West, J. Appl. Lett. 69, 6517 (1991).

${ }^{5}$ B. F. Levine, A. Zussman, J. M. Kuo, and J. de Jong, J. Appl. Lett. 71, 5130 (1992).

${ }^{6}$ B. F. Levine, C. G. Bethea, G. Hasnain, J. Walker, R. J. Malik, and J. M. Vandenberg, Phys. Rev. Lett. 63, 899 (1989).

${ }^{7}$ M. A. Kinch and A. Yariv, Appl. Phys. Lett. 55, 2093 (1989).

${ }^{8}$ G. Destefanis, P. Audebert, E. Mottin, and P. Rambaud, J. Cryst. Growth 185, 1288 (1998)

${ }^{9}$ L. C. West and S. J. English, Appl. Phys. Lett. 46, 1156 (1985).

${ }^{10}$ J. Y. Andersson, L. Lundqvist, and Z. F. Paska, Appl. Phys. Lett. 58, 2264 (1991).

${ }^{11}$ R. P. G. Karunasiri, J. S. Park, J. Chen, and R. Shih, Appl. Phys. Lett. 67, 2600 (1995).

${ }^{12}$ E. H. Li, Quantum Well Mixing and Optoelectronic Device Applications, Milestone Series Vol. 145 (WA, SPIE, Bellingham, 1998).

${ }^{13}$ J. D. Ralston, M. Ramsteiner, B. Discher, M. Maier, G. Brandt, P. Koidl, and D. J. As, J. Appl. Phys. 70, 2195 (1991).

${ }^{14}$ A. G. Steele, M. Buchanan, H. C. Liu, and Z. R. Wasilewski, J. Appl. Phys. 75, 8234 (1994).

${ }^{15}$ J. I. Malin, P. Liu, D. K. Sengupta, W. C. Fang, S. L. Chuang, M. Feng, G. E. Stillman, and K. C. Hsieh, J. Appl. Phys. 80, 4737 (1996).

${ }^{16} \mathrm{~J}$. Crank, The Mathematics of Diffusion, 2nd ed. (Oxford University Press, Oxford, 1975), p. 15.

${ }^{17}$ H. Asai and K. Oe, J. Appl. Phys. 54, 2052 (1983).

${ }^{18}$ B. Elman, E. S. Koteles, P. Melman, C. Jagannath, C. A. Armiento, and M. Rothman, J. Appl. Phys. 68, 1351 (1990).

${ }^{19}$ S. Bürker, M. Baeumler, J. Wanger, E. C. Larkins, W. Rothemund, and J. D. Ralston, J. Appl. Phys. 79, 6818 (1996).

${ }^{20}$ L. H. Peng and C. G. Fonstad, J. Appl. Phys. 77, 747 (1995).

${ }^{21}$ L. H. Peng, J. H. Smet, T. P. E. Broekaert, and C. G. Fonstad, Appl. Phys. Lett. 61, 2078 (1992).

${ }^{22}$ D. G. Deppe and N. Holonyak, Jr., J. Appl. Phys. 64, R93 (1988).

${ }^{23}$ B. F. Levine, A. Zussman, S. D. Gunapala, M. T. Asom, J. M. Kuo, and W. S. Hobson, Appl. Phys. Lett. 72, 4429 (1992).

${ }^{24}$ H. C. Liu, Z. R. Wasilewski, M. Buchanan, and Hanyou Chu, Appl. Phys. Lett. 63, 761 (1993).

${ }^{25}$ A. S. W. Lee and E. H. Li, Appl. Phys. Lett. 69, 3581 (1996).

${ }^{26}$ G. M. William, R. E. DeWames, C. W. Farley, and R. J. Anderson, Appl. Phys. Lett. 60, 1324 (1992).

${ }^{27}$ B. F. Lavine, R. J. Malik, J. Walker, K. K. Choi, C. G. Bethea, D. A. Kleinman, and J. M. Vandenberg, Appl. Phys. Lett. 50, 273 (1987).

${ }^{28}$ K. M. S. V. Bandara, B. F. Levine, and M. T. Asom, J. Appl. Phys. 74, 346 (1993).

${ }^{29}$ K. K. Choi, M. Taysing-Lara, P. G. Newman, and W. Chang, Appl. Phys. Lett. 61, 1781 (1992).

${ }^{30}$ G. Hasnain, B. F. Levine, C. G. Bethea, R. R. Abbott, and S. J. Hsieh, J. Appl. Lett. 67, 4361 (1990).

${ }^{31}$ A. Harwit and J. S. Harris, Jr., Appl. Phys. Lett. 50, 685 (1987).

${ }^{32}$ S. D. Gunapala, K. M. S. V. Bandara, B. F. Levine, G. Sarusi, D. L. Sivco, and A. Y. Cho, Appl. Phys. Lett. 64, 2288 (1994).

${ }^{33}$ K. K. Choi, J. Appl. Phys. 73, 5230 (1993). 